\title{
Topical morphine for treatment of cancer-related painful mucosal and cutaneous lesions: a double-blind, placebo-controlled cross-over clinical trial
}

\author{
Aleksandra Ciałkowska-Rysz, Tomasz Dzierżanowski
}

Paliative Care Unit, Oncology Chair, Medical University of Lodz, Lodz, Poland

Submitted: 30 May 2017

Accepted: 17 July 2017

Arch Med Sci 2019; 15, 1: 146-151

DOI: https://doi.org/10.5114/aoms.2018.72566

Copyright @ 2018 Termedia \& Banach

\section{Abstract}

Introduction: Painful mucosal and cutaneous lesions are often less responsive or even refractory to systemic opioid analgesics. There is evidence suggesting that the effectiveness of topical morphine be restricted to inflammatory pain. The studied groups were small and the observation period relatively short. The aim of this study was to assess the effectiveness and safety of topical morphine for pain related to mucosal lesions and skin ulcers.

Material and methods: The study was a 14-day randomized placebo-controlled cross-over trial (RCT) with a 28-day follow-up open phase (OP). The trial was conducted in adult patients with localized cancer-related pain and treated with systemic opioids in an oncology center or home hospice. The patients administered $0.2 \%$ gel on the mucosal lesion or $0.2 \%$ ointment on the skin lesion by themselves, without restrictions regarding the number of doses per day. The primary measurements were mean pain intensity (MPI) and mean pain relief (MPR) on the numeric rating scale (NRS 0-10), and ITT analysis was performed.

Results: Thirty-five patients were randomized to the RCT, and all of them completed 14-day observation. The MPI before the treatment was NRS 5.9 and decreased to 2.5 after morphine ( $p<0.0001$ vs. placebo). The MPR was $57 \%$ after morphine, and $77 \%$ of the patients using topical morphine obtained clinically significant (at least $50 \%$ of the starting value) pain relief, statistically different from placebo. The analgesic effect was sustained over the 28-day OP period ( $p=0.00001)$. There were only 2 cases of moderate pruritus, and no other side effects were reported.

Conclusions: Topical morphine was found to be a fast acting, highly effective, and safe medication for mucosal and skin lesions in palliative patients, with a sustainable pain relief effect over the 28-day observation period.

Key words: topical morphine, cancer pain, mucosal lesions, cutaneous lesions.

\section{Introduction}

Painful mucosal and cutaneous lesions are often less responsive or even refractory to systemic opioid analgesics and may result in increases of doses. Topical opioids proved to relieve inflammatory pain without systemic adverse events in such cases [1]. Mu-opioid receptors are activated in the inflammatory process in human epidermis and keratinocytes [2]. There are a number practices, formulations, and dose concen-

\author{
Corresponding author: \\ Tomasz Dzierżanowski \\ MD, PhD \\ Paliative Care Unit \\ Oncology Chair \\ Medical University \\ of Lodz \\ 2 Ciołkowskiego St \\ 93-510 Lodz, Poland \\ Phone: +48 601334001 \\ E-mail: t.dzierzanowski@ \\ termedia.pl
}


trations of the topical preparations [3]. There has also been growing evidence since the 1990s that they are efficient and safe in acute and chronic pain conditions. However, their use remains unpopular so far [4].

The use of topical morphine seems to be restricted to inflammatory pain. In a randomized controlled trial, topical morphine sulfate was found not to be as effective when used for the pain associated with superficial burns as when used for the pain associated with chronic inflammatory wounds [5]. It did not relieve pain either during topical photodynamic therapy or in sunburn wounds [6]. Neuropathic cancer pain does not respond to topical morphine [7]. In a short randomized, placebo-controlled, crossover study of topically applied morphine as a treatment for painful leg ulcers, there was a statistically significant difference only $2 \mathrm{~h}$ after dressing on the first treatment occasion [8]. In another trial the duration of pain relief after morphine mouthwash in patients with radiotherapy- and chemotherapy-induced oral mucositis was around $120 \min [9]$.

The aim of this study was to assess the effectiveness and safety of topical morphine for the treatment of pain caused by mucosal lesions $(0.2 \%$ hydrogel) and skin ulcers ( $0.2 \%$ ointment) in comparison to placebo in adult cancer patients treated with systemic opioids. The patients administered the gel or the ointment on the lesion by themselves, without restrictions regarding the number of doses per day. The hydrogel and the ointment were prepared in the hospital pharmacy according to the following prescriptions:

\begin{tabular}{|c|c|c|}
\hline \multicolumn{3}{|c|}{ Morphine ointment $0.2 \%$ : } \\
\hline Rp: & Morphine sulfate & $0.2 \mathrm{~g}$ \\
\hline & Glycerol & $3.0 \xi$ \\
\hline & $\begin{array}{l}\text { Eucerin } \\
\text { Mixf ung }\end{array}$ & up to $100.0 \mathrm{~g}$ \\
\hline
\end{tabular}

Morphine gel $0.2 \%$ :

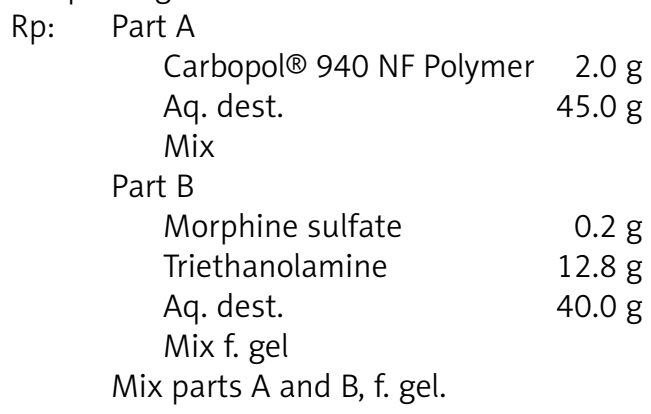

The prescriptions were worked out based on the results of a detailed study on the effect of cross-linking based on the properties of hydrogels with Carbopol and on pharmaceutical availability of morphine sulfate [10].

\section{Material and methods}

The study consisted of two phases: a 14-day randomized placebo-controlled cross-over trial and a 28-day follow-up open trial.

The inclusion criteria were:

1. Adults (18 years and more).

2. Signed informed consent.

3. Localized cancer-related pain of the intensity at least NRS 4.

4. Systemic opioid or non-opioid analgesic doses stable for the past 7 days.

The exclusion criteria were:

1. Chemotherapy or radiotherapy in the past 1 month before the study.

2. Local skin or mucosa infection.

3. Local irritation or other side effects related to application of the gel. However, if mild adverse effects appeared during the treatment, the study continued on the patient's request.

The patients were randomized using computer-generated randomization numbers.

The randomized double-blinded cross-over study lasted 14 days. On day 0, after providing written consent, patients were recruited and randomized (computer generated random sequence) to two subgroups: treated with morphine first (group M) and receiving placebo first (group P). After 7 days there was a switch of treatment.

The assessment of pain intensity was performed on days 0,7 and 14 .

The patients were offered to continue the treatment in an open phase and received morphine for the next 28 days. The aim of the open phase was to assess the sustainability of the analgesic effect of the topical treatment and to register late side effects. The pain intensity was assessed on day 28. As a starting pain intensity score, we assumed the value on day 7 in group $M$ and day 14 in group $P$.

The primary outcome measures for the randomized study were:

1. Pain intensity in subgroups treated with morphine versus placebo, on the numeric rating scale (0-10) (NRS). The assessment took place on days 7 and 14 .

2. Pain relief (the difference between the starting NRS value and the values after morphine treatment and placebo). A positive value means a decrease in pain intensity.

The secondary measure was pain intensity on day 42 (28 of the open phase) versus the starting value. The starting value was pain intensity after morphine administration assessed on day 7 in the $M$ subgroup and day 14 in the $P$ subgroup. The intention was to test the sustainability of the analgesic effect.

On the final day (14) of the randomized study, the patients were assessed and proposed to continue the treatment with the gel containing the ac- 
tive agent. In the case of side effects, the patients were not included in the open phase.

The measures for the open study were:

1. The number and severity of side effects.

2. The sustainability of the analgesic effect over a 28-day period, counted as the difference between the starting value (after morphine treatment assessed appropriately on day 7 or 14 of the randomized study) and the final value on day 28 of the open phase). No statistical difference proves the sustainability of the treatment.

The trial was conducted in Stowarzyszenie Hospicjum Łódzkie (hospice) in 2009-2013 in Lodz. The ethical committee of the Medical University of Lodz approved the study (decision RNN/194/06/ KE of Nov. 28, 2006).

\section{Statistical analysis}

McNemar's test was used to compare proportions of nominal data between subgroups (men-women, young-old). The Wilcoxon signedrank test was used to compare pain relief after

Table I. Characteristics of the studied population

\begin{tabular}{|c|c|}
\hline Parameter & Value \\
\hline Total number of patients: & 35 \\
\hline Women & 22 \\
\hline Average age [years] & 61.6 \\
\hline Primary diagnosis: & Patients \\
\hline C53 cervix & 8 \\
\hline C50 breast & 8 \\
\hline C20 rectum & 5 \\
\hline C32 larynx & 3 \\
\hline C43 melanoma & 3 \\
\hline C80 prime focus unknown & 2 \\
\hline C05 palate & 2 \\
\hline $\begin{array}{l}\text { C82 lymphoma, C } 44 \text { skin, C34 lung, } \\
\text { C08 salivary gland }\end{array}$ & 1 (each) \\
\hline Region affected by pain & Patients \\
\hline Crotch & 8 \\
\hline Chest & 6 \\
\hline Sacrum & 5 \\
\hline Anus & 5 \\
\hline Neck & 3 \\
\hline Parotid region & 2 \\
\hline Back & 2 \\
\hline Submandibular region, shin, nose, trunk & 1 (each) \\
\hline
\end{tabular}

morphine versus placebo treatment. The KruskalWallis one-way analysis of variance was applied for the analysis of pain intensity. $P$-values less than 0.05 were considered statistically significant.

The estimated sample size with the statistical power of $80 \%$ was 10 patients. However, due to the intention of long-term observation in the open phase, the planned number of studied patients was 25 .

The intention-to-treat (ITT) approach was used to avoid the bias associated with the non-random loss of participants

\section{Results}

\section{Characteristics of population}

Thirty-five patients were included in the study, signed written consent and underwent randomization. There were no exclusions during the treatment period, and all the patients completed the trial. The characteristics of the studied population regarding primary diagnosis and region affected by pain are presented in Table I. Twenty-two (63\%) patients had skin lesions and the rest (37\%) both mucositis and skin lesions.

There were 17 patients in group $M$ (morphine first) and 18 in group $P$ (placebo first). The average age was 61.1 and 62.1 years respectively, and the number of women in each subgroup was 11 . The groups did not differ statistically from each other in terms of sex ( $p=0.83$; McNemar's test) and age $(p=0.84$; $t$-test). There was no difference in the number of patients treated with weak and strong systemic opioids between the groups either ( $p=$ 0.51 ; Kruskal-Wallis test).

On day 14 of the randomized cross-over study, 33 patients entered the 28-day long open study phase, with the active topical treatment.

The flow of the patients and the pain intensity results are presented in Figure 1.

\section{Pain intensity}

The mean pain intensity (MPI) on day 0 was 5.9 (95\% Cl: 5.1-6.7; range: $4-8)$ on the NRS (0-10) in both $\mathrm{P}$ and $M$ subgroups ( $p=0.99$ ), with no difference between patients receiving systemic opioid treatment and non-opioid analgesics.

On day 7, the MPI was 4.6 (95\% Cl: 3.3-5.9) and 2.5 (95\% Cl: $1.6-3.3)$ in $P$ and $M$ subgroups respectively. On day 14 , the $\mathrm{MPI}$ was $2.5(95 \% \mathrm{Cl}$ : 1.6-3.4) and $5.2(95 \% \mathrm{Cl}: 4.4-6.1)$ in $\mathrm{P}$ and $M$ subgroups respectively (Figure 2 ). The differences were statistically significant $(p<0.0001)$.

\section{Pain relief}

On the days 7 and 14 pain intensity was reassessed in each patient. The mean pain relief was 


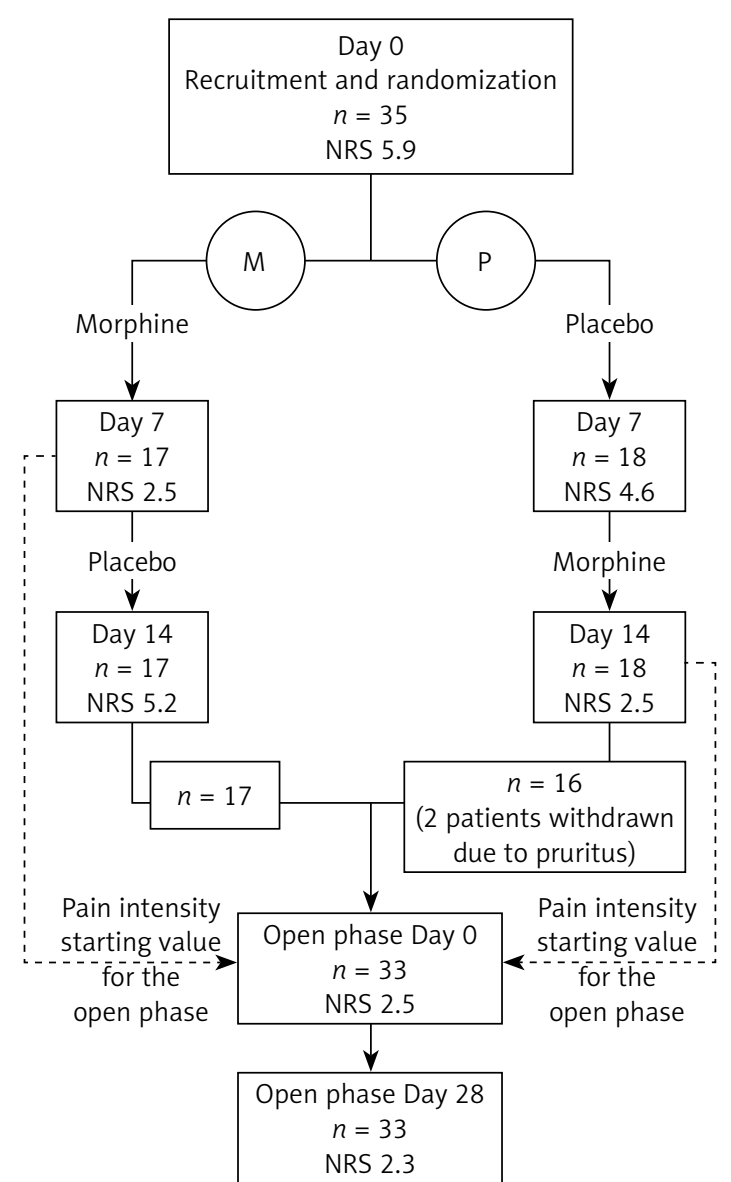

Figure 1. Flow of the trial

57\% (range: 40-100\%) after morphine treatment and $16 \%$ (range: $0-50 \%$ ) after placebo (Figure 3), and there was a statistically significant difference between the treatments in favor of morphine ( $p=$ 0.0000004 in the Wilcoxon $T$ test). Twenty-seven (77\%) patients using topical morphine obtained clinically significant (at least $50 \%$ of the starting value) pain relief. Significant pain relief on day 7 was observed in $76 \%$ (14) and $17 \%$ (3) of patients receiving morphine and placebo respectively (number needed to treat 1.67).

The difference for morphine versus placebo was also statistically significant in all subgroups of systemic treatment (non-opioid, weak opioid and strong opioid subgroups). There was no difference in pain relief after morphine between these subgroups $(p=0.86)$.

\section{Sustainability of pain relief}

In subgroup M, the MPI was $5.2(95 \% \mathrm{Cl}$ : $4.4-$ 6.1) on the NRS (0-10) on day 0 and $2.3(95 \% \mathrm{Cl}$ : 1.6-3.7) on day 28 of the open phase. The improvement was expected, as in this group the treatment with topical morphine was restarted.

In subgroup P, the MPI was 2.5 (95\% Cl: $1.6-$ 3.4) and $2.4(95 \% \mathrm{Cl}: 1.7-3.7)$ on days 0 and 28 respectively.

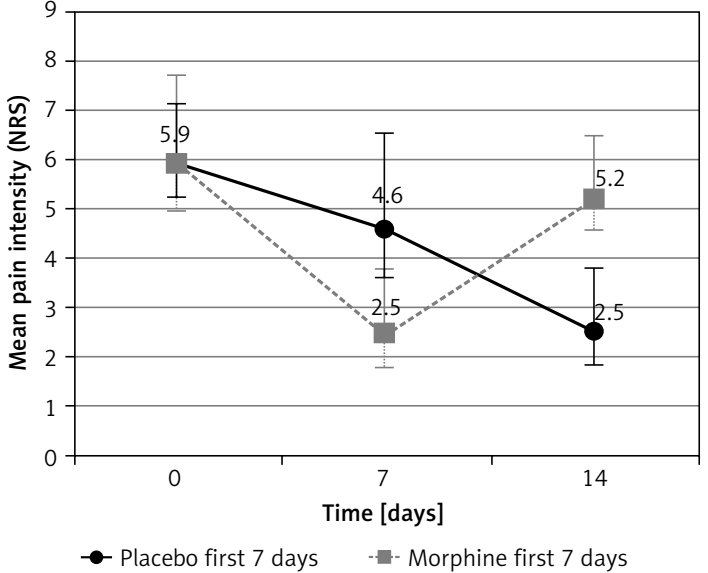

Figure 2. Mean pain intensity during cross-over phase (95\% confidence intervals)

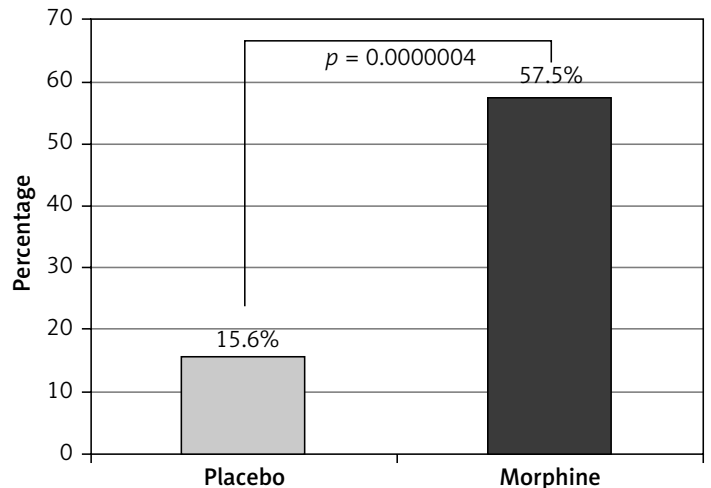

Figure 3. Pain relief after 7 days of topical morphine administration

In the whole group, the mean pain intensity on day 28 of the open phase was $2.3(95 \% \mathrm{Cl}$ : 1.6$3.5)$ on the NRS (0-10) and was significantly lower than the starting value $(p=0.00001)$. It did not differ from the starting value on the last day of active treatment (NRS 2.5; $p=0.068$ ).

\section{Safety}

All 35 patients completed the 14-day study. The only side effect was moderate pruritus that was reported by 2 patients on day 14 and was related to the administration of topical morphine. Both the patients decided not to enter the open study phase. No side effects were reported in the 28-day open study phase. No systemic side effects were reported in either phase of the trial.

\section{Discussion}

This study aimed to assess the effectiveness of topically administered morphine strictly in cancer-related painful mucosal and cutaneous lesions. In one randomized, double-blind, placebo-controlled, crossover pilot study of 5 patients 
with painful sacral sores, morphine applied topically to painful ulcers was found to be an effective and well-tolerated method of analgesia, and was not associated with systemic adverse effects [11]. Similar effects were observed in a 48-hour observation of 16 patients with bedsores and cancer sores [12]. There are also some case reports and trials with small numbers of patients on the effectiveness of diamorphine [13, 14]. In a study with 13 palliative care patients diamorphine was found to be an effective treatment for pain caused by stage II or III pressure ulcers, and as safe as placebo, but a larger study was supposed to be performed to confirm these results [15].

In this randomized, double-blind, placebo-controlled, crossover trial, 35 patients were enrolled and finished the study. Its incremental advantage seems to be the assessment of both skin and mucosal lesions and much bigger studied group, compared to the previous evidence. Mucosal lesions are infrequent but refractory to systemic analgesia, painful conditions. $0.2 \%$ ointment for skin ulcers and $0.2 \%$ hydrogel on mucosa were found to be simple and unproblematic for the patients to use. The studied group seems to be typical for the palliative care population.

The number of patients enrolled in the study exceeded that planned to ensure the statistical power of the trial. The subgroups did not differ from each other. In these terms, the trial met the expectations of the authors.

The mean pain intensity at the starting day was NRS 5.9, regardless of whether the systemic opioid treatment was provided or not. Both ointment and hydrogel were found to be efficient, and the end mean pain intensity of NRS 2.5 (not exceeding NRS 4) was not only statistically significant but also an excellent clinical response.

The effect remained unchanged or even slightly improved over the next 28 days of the open-label trial. Intentionally we compared the end day effect to the value on the last day of the active treatment. It is probably the first long-term observation of topical opioid agents in cancer patients. The sustainability of the effect might be the result of a proper and uncomplicated way of administration. However, the daily dose of the medication was not tracked in the study, and the patients were allowed to use it as frequently as necessary, by covering all the painful lesions. Even though topical morphine appeared to be well tolerated, 2 patients ended the observation on the $14^{\text {th }}$ day due to moderate itching. However, they were discouraged from entering the treatment in the open-label phase by the investigator.

The pain relief not only was a long-lasting effect but also it appeared quickly. Seventy-six percent of patients treated with morphine reported clinically significant (50\%) pain relief on day 7 , versus $17 \%$ of the control group. The number needed to treat of 1.67 on day 7 proves the high effectiveness of topical morphine.

The concentration of morphine was $0.2 \%$ both in the ointment and in the hydrogel in this study. Different concentrations have been investigated with success by other researchers (up to $2 \%$ ) [16].

A limitation of this study is the lack of information on morphine daily dose administered topically and the reference to the systemically administered opioid doses. Although this is probably the largest study on topically administered morphine, it is still too small to perform a multivariable analysis as well.

In conclusion, topical morphine was found to be a fast acting, highly effective, and safe medication for mucosal and skin lesions in palliative patients, with a sustainable pain relief effect over the long-term observation period.

\section{Acknowledgments}

This research received no specific grant from any funding agency, public or commercial, or from non-profit sectors, and was fully covered by the University. We declare no financial relationship with any organizations or industry.

\section{Conflict of interest}

The authors declare no conflict of interest.

\section{References}

1. Stein C. Peripheral mechanisms of opioid analgesia. Anesth Analg 1993; 76: 182-91.

2. Bigliardi PL, Bigliardi-Qi M, Buechner S, Rufli T. Expression of mu-opiate receptor in human epidermis and keratinocytes. J Invest Dermatol 1998; 111: 297-301.

3. Graham T, Grocott P, Probst S, Wanklyn S, Dawson J, Gethin G. How are topical opioids used to manage painful cutaneous lesions in palliative care? A critical review. Pain 2013; 154: 1920-8.

4. Stein C. The control of pain in peripheral tissue by opioids. N Engl J Med 1995; 332: 1685-90.

5. Welling A. A randomised controlled trial to test the analgesic efficacy of topical morphine on minor superficial and partial thickness burns in accident and emergency departments. Emerg Med J 2007; 24: 408-12.

6. Draxler J, Schuch M, Paul A, et al. Topical application of morphine and buprenorphine gel has no effect in the sunburn model [German]. Schmerz 2008; 22: 571-4.

7. Mercadante S, Portenoy RK. Opioid poorly-responsive cancer pain. Part 1: clinical considerations. J Pain Symptom Manage 2001; 21: 144-50.

8. Bastami S, Frödin T, Ahlner J, Uppugunduri S. Topical morphine gel in the treatment of painful leg ulcers, a double-blind, placebo-controlled clinical trial: a pilot study. Int Wound J 2012; 9: 419-27.

9. Vayne-Bossert P, Escher M, de Vautibault CG, et al. Effect of topical morphine (mouthwash) on oral pain due to chemotherapy- and/or radiotherapy-induced mucositis: a randomized double-blinded study. J Palliat Med 2010; 13: 125-8. 
10. Samczewska G, Kołodziejska J, Ciałkowska-Rysz A, Piechota-Urbańska M. The effect of cross-linking base on the properties of hydrogels with Carbopol and on pharmaceutical availability of morphine sulphate. Arch Med Sci 2007; 3: 19-26.

11. Zeppetella G, Paul J, Ribeiro MD. Analgesic efficacy of morphine applied topically to painful ulcers. J Pain Symptom Manage 2003; 25: 555-8.

12. Zeppetella G, Ribeiro MD. Morphine in intrasite gel applied topically to painful ulcers. J Pain Symptom Manage 2005; 29: 118-9.

13. Flock P, Gibbs L, Sykes N. Diamorphine-metronidazole gel effective for treatment of painful infected leg ulcers. J Pain Symptom Manage 2000; 20: 396-7.

14. Porzio G, Aielli F, Verna L, Cannita K, Marchetti P, Ficorella $C$. Topical morphine in the treatment of painful ulcers. J Pain Symptom Manage 2005; 30: 304-5.

15. Flock P. Pilot study to determine the effectiveness of diamorphine gel to control pressure ulcer pain. J Pain Symptom Manage 2003; 25: 547-54.

16. Sarvizadeh M, Hemati S, Meidani M, Ashouri M, Roayaei M, Shahsanai A. Morphine mouthwash for the management of oral mucositis in patients with head and neck cancer. Adv Biomed Res 2015; 4: 44. 